\title{
Primary Care and Pre-exposure Prophylaxis Services in Publicly Funded Family Planning Clinics in the Southern United States
}

\author{
Caroline G. Coleman, $M D^{7}$, Jessica M. Sales, PhD², Cam Escoffery, PhD',

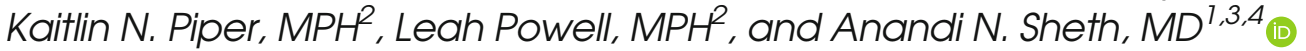

${ }^{1}$ School of Medicine, Emory University, Atlanta, GA, USA; ${ }^{2}$ Department of Behavioral Sciences and Health Education, Rollins School of Public Health, Emory University, Atlanta, GA, USA; ${ }^{3}$ Department of Medicine, Division of Infectious Diseases, Emory University School of Medicine, Atlanta, GA, USA; ${ }^{4}$ Grady Health System, Atlanta, GA, USA.

BACKGROUND: HIV pre-exposure prophylaxis (PrEP) is underutilized by US women. Cost and resource concerns are barriers to PrEP delivery in settings that see men. Family planning clinics may be ideal PrEP delivery settings for women, but as they are not uniform in their clinical services, cost and resource concerns may vary.

OBJECTIVE: We examined factors that influence perceptions of costs and resources related to PrEP delivery in Title X-funded family planning clinics in Southern states, which overlaps with high HIV-burden areas.

DESIGN: We conducted a web-based survey among a convenience sample of clinicians and administrators of Title $\mathrm{X}$ clinics across 18 Southern states (DHHS regions III, IV, VI). We compared cost- and resource-related survey items and other clinic- and county-level variables between clinics by whether their clinics also provided other primary care services. We analyzed interviews for cost and resource themes.

PARTICIPANTS: Title X clinic staff in the South.

KEY RESULTS: Among 283 unique clinics, a greater proportion of clinics that also provided primary care currently provided PrEP compared with those that did not provide primary care $(27.8 \%$ vs. $18.3 \%, p=0.06)$, but this difference was not statistically significant. Among 414 respondents in clinics that were not providing PrEP, those in clinics with primary care services were more likely to respond that they had the necessary financial resources ( $p$ $<0.01)$ and staffing $(p<0.01)$ for PrEP implementation compared to those without primary care services. In interviews, respondents differed on concerns about costs of labs and staffing based on whether their clinic had concomitant primary care services or not.

CONCLUSIONS: Among publicly funded Southern family planning clinics, current PrEP provision was higher among clinics with concomitant primary care. Among clinics not providing PrEP, those with concomitant primary care services have lower perceived cost and resource barriers and therefore may be optimal for expanding PrEP among women.

Prior Presentations This manuscript was presented in part at the InterCFAR HIV in Women Symposium, Chicago, IL, October 21-22, 2019 (poster presentation).

Received August 7, 2020

Accepted December 17, 2020

Published online January 14, 2021
KEY WORDS: PrEP; family planning; HIV; women's health; Title X.

J Gen Intern Med 36(10):2958-65

DOI: $10.1007 / \mathrm{s} 11606-020-06509-3$

(C) Society of General Internal Medicine 2021

\section{INTRODUCTION}

In 2018, women comprised almost $20 \%$ of the nearly 40,000 new HIV infections in the USA every year, with Southern states accounting for a disproportionate number. ${ }^{1,2}$ Effective prevention efforts tailored to women are therefore urgently needed. HIV pre-exposure prophylaxis (PrEP) is a once daily pill that is recommended for people who are at risk for acquiring HIV. When accounting for adherence, PrEP is at least $90 \%$ effective in reducing HIV acquisition in women, yet it remains underutilized among women. ${ }^{3}$ The few available studies on US women report low PrEP knowledge, awareness, and use among women, particularly women in the South. ${ }^{46}$ Family planning clinics are of particular interest as potential PrEP delivery sites, as they are already trusted and accessed routinely by women: $60 \%$ of young women utilize family planning for sexual health and preventative services. ${ }^{7}$

Despite these attractive factors, challenges exist for PrEP implementation in family planning clinics. ${ }^{8}$ In clinical settings that see mostly men, cost concerns are common barriers to PrEP delivery. Costs of PrEP prevision include not only cost of the medication itself, but also the pre-implementation costs (personnel and supply costs of planning, training) and implementation costs (personnel costs of staff and providers; laboratory costs for testing for HIV, pregnancy, creatinine, hepatitis $\mathrm{B}$, and sexually transmitted infections; lost revenue due to longer visits with fewer overall patients) for the clinic to provide PrEP. In a 2015 survey of providers, $36 \%$ were less willing to prescribe PrEP due to worries about cost and unclear insurance coverage. ${ }^{9}$ In fact, uninsured patients are four times less likely to use PrEP compared to insured ones. ${ }^{10}$

Among family planning clinics, publicly funded family planning clinics are of even more interest as they are safety-net providers, particularly in regions without Medicaid expansion, like many Southern states. ${ }^{11}$ The Title X Family Planning 
Program, administered by the U.S. Office of Population Affairs, provides grants to health department-or county hospital-based programs, specialized family planning clinics, federally qualified health centers (FQHCs), and other community health clinics for the delivery of comprehensive family planning and preventive health services, especially for low-income individuals. However, as nearly half of family planning patients seen at Title $\mathrm{X}$ clinics in the South are uninsured and recent restrictions have reduced the number of clinics within the Title X network, cost concerns may be even more pertinent for these clinics. ${ }^{12,13}$ Notably, Title $\mathrm{X}$-funded family planning clinics are not uniform in the services they provide, as some only provide family planning and sexual healthcare, but others integrate family planning within primary care services (e.g., FQHCs). Primary care is an ideal site for PrEP delivery, as these settings already possess the infrastructure for long-term health maintenance, and may require less intervention and support to begin offering PrEP. Thus, clinic cost- and resource-related concerns may vary based on clinic services provided.

In our recent survey of administrators and clinicians working in Title X clinics in the South, we found a significant positive association between readiness for PrEP implementation and general assessment of resources. ${ }^{14}$ Thus, the overall objective of this analysis was to assess factors that influence the perceptions of costs and resources associated with PrEP provision in Title X family planning clinics in the South. We hypothesized that cost and resource perceptions would vary by a clinic's provision of other primary care services.

\section{MATERIALS AND METHODS}

\section{Study Design}

We used previously collected survey and interview data from an explanatory, sequential mixed methods research study focused on readiness for PrEP implementation in Title X clinics in the South. ${ }^{15}$ Quantitative surveys were administered online in February through June 2018 and were followed up by key informant interviews of a subset of survey respondents that were purposively sampled to ensure representation across regions, states, and clinic roles, and by their clinic's current PrEP provision, conducted between March and July 2018. In order to understand factors associated with readiness to implement PrEP, the parent study surveyed perceptions of costs and resources required for PrEP implementation only among respondents from clinics that had not yet implemented PrEP. ${ }^{16}$ As preliminary quantitative analyses from the survey suggested key differences in perceptions of cost and resources required for PrEP implementation based on the clinical services offered, we subsequently analyzed interviews of respondents from non-PrEP-providing clinics to better understand cost- and resource-related barriers to implementation in this setting. Approval was obtained from the Emory University and University of North Carolina Institutional Review Boards.

\section{Study Population and Recruitment}

Web-based quantitative surveys were conducted as previously described. ${ }^{11}$ In brief, healthcare providers and clinic administrators from Title $\mathrm{X}$-funded family planning clinics in the Department of Health and Human Services regions III (midAtlantic states), IV (Southeastern states), and VI (Southwestern states) were invited to participant in the online survey and follow-up qualitative interviews. A total of 1782 Title X clinics are located in these regions, which overlap with high HIVburden areas and collectively make up the Southern US. Recruitment occurred via the National Clinical Training Center for Family Planning (NCTCFP) listserv emails, advertisement on the NCTCFP website, engagement with state Title $\mathrm{X}$ grantees, and in-person recruitment at the NCTCFP national meeting.

\section{Survey and Interview Guide Development}

The quantitative survey utilized the Consolidated Framework for Implementation Research (CFIR), which provides a menu of constructs that can be used as a practical guide for systematically assessing potential facilitators and barriers in preparation for implementing a new evidence-based practice. ${ }^{17}$ The 93-item survey assessed a total of 11 CFIR implementationfocused constructs based on a review of US-based PrEP implementation literature. All CFIR-related survey items were evaluated on a 5 -point Likert scale $(1=$ strongly disagree to 5 $=$ strongly agree).

Qualitative interviews were semi-structured with prompts based on CFIR constructs. The interview guide consisted of 8 primary domains: HIV Priority, PrEP Priority, Capacity and Implementation, Resources, Adoption and Decision Making around New Practices, Champions, Trainings, and External Factors. Interviews were conducted by trained research staff; transcripts were recorded and professionally transcribed verbatim. We created a codebook using CFIR, coded transcripts, and then employed NVivo to extract CFIR themes. To further explore quantitative findings in this group, we only utilized data from interview participants from clinics that did not provide PrEP for this analysis.

\section{Measures}

Clinic-level characteristics assessed in the survey included clinic type (FQHC, hospital or health department, other), staff to provide insurance support onsite (yes, no), pharmacy onsite (yes, no), and services provided by clinic (family planning only, family planning and primary care), metropolitan location (yes, no), and DHHS region (III, IV, VI). We also captured characteristics of the county where the clinic was located using US Census and AIDSVu data. ${ }^{18}$ County-level characteristics included as follows: percent of population under $200 \%$ of the federal poverty level, percent uninsured, percent with high school education, percent in urban area, median income, percent White, and percent Hispanic. Since there were multiple respondents from some clinics, unique clinics were identified 
by unique addresses, and a unique clinic dataset was created where the most common response to clinic-level items answered by participants within a clinic (e.g., clinic type) was used to summarize clinic- and county-level variables.

Since cost considerations for PrEP delivery include both cost of the intervention and clinic resources dedicated for implementation, our evaluated outcomes included 10 total survey items in the Cost and Available Resources (PrEPspecific Funding and General Assessment) CFIR domains (Table 2). These outcomes were surveyed only among respondents from clinics that did not provide PrEP.

\section{Data Analyses}

Quantitative analyses were conducted using SAS version 9.4 (Cary, NC). Descriptive statistics were performed on clinic- and county-level characteristics and CFIR survey items (means (SD) or counts (\%) for all respondents and/ or clinics, where appropriate). For the question related to whether a respondent's clinic provided PrEP or primary care services, responses of "unknown" were combined with "no." No other clinic-level variables allow for "unknown" as a response, and missingness was rare across all clinic-level variables (less than $1 \%$ missing for each variable). Composite scores for each CFIR construct were calculated by taking the average of the contributing survey items. We used unpaired $t$ tests, chi-square tests, or Fisher's exact test where appropriate, for comparisons by primary care provision status. Fisher's exact tests were used instead of chi-square tests when the values in any of the cells were less than 5 .
For qualitative analysis, interviews were imported into NVivo 12. The study team used standard qualitative data analysis methods including reading of transcripts, creation of a codebook, double coding, and consensus meetings. ${ }^{19}$ Qualitative rigor and coding consistency was established through a team coding approach. Multiple team members met to develop a codebook based on topics covered in the interview guide. The codebook contained code definitions, inclusion/exclusion criteria, and example quotes. Using this codebook, at least two analysts coded each transcript. The analysts first coded transcripts independently; then, they met to compare their coding and resolve any discrepancies through discussion. All transcripts were coded until $100 \%$ agreement was reached between the analysts. For this analysis, we analyzed interviews from non-PrEP-providing clinics to better understand barriers to implementation in this setting, as the vast majority of clinics we studied had not yet implemented PrEP. We extracted the "resources" code from NVivo and then assessed the theme by clinic primary care provision status.

\section{RESULTS}

\section{Quantitative Results}

Description of Unique Clinics Overall and by Primary Care Provision Status. In our convenience study, respondents from 283 clinics of the 1782 total clinics in DHHS regions II, III, or VI participated (15.9\%). The 283 unique clinics were represented by 519 survey respondents who completed the survey from an eligible DHHS region; $20.2 \%$ clinics $(n=$ $60)$ were currently providing PrEP. The proportion of clinics

Table 1 Descriptive Statistics of Unique Non-PrEP-Providing Clinics, Stratified by Provision of Primary Care Services $(n=223)$

\begin{tabular}{|c|c|c|c|c|}
\hline \multirow[b]{2}{*}{ Variable } & \multicolumn{4}{|c|}{ Counts (\%) or mean (SD) } \\
\hline & $\begin{array}{l}\text { Primary care } \\
N=65\end{array}$ & $\begin{array}{l}\text { No primary care } \\
N=158\end{array}$ & $p$ value $^{\mathrm{a}}$ & $\begin{array}{l}\text { Total } \\
N=223\end{array}$ \\
\hline \multicolumn{5}{|l|}{ Clinic-level characteristics } \\
\hline \multicolumn{5}{|l|}{ Clinic classification: } \\
\hline Federally qualified health center & $19(29.7 \%)$ & $2(1.3 \%)$ & $<0.0001$ & $21(9.5 \%)$ \\
\hline Health department-based clinic & $36(56.2 \%)$ & $143(90.5 \%)$ & & $179(80.6 \%)$ \\
\hline Other family planning clinic type & $9(14.1 \%)$ & $13(8.2 \%)$ & & $22(9.9 \%)$ \\
\hline Insurance support onsite & $52(80.0 \%)$ & $101(63.9 \%)$ & 0.019 & $153(68.6 \%)$ \\
\hline Pharmacy onsite & $27(41.5 \%)$ & $61(39.1 \%)$ & 0.74 & $88(39.2 \%)$ \\
\hline \multicolumn{5}{|c|}{ Department of Health and Human Services region: } \\
\hline Region 3 (mid-Atlantic) & $15(23.1 \%)$ & $43(27.2 \%)$ & 0.19 & $58(26.0 \%)$ \\
\hline Region 4 (Southeastern) & $44(67.7 \%)$ & $88(55.7 \%)$ & & $132(59.2 \%)$ \\
\hline Region 6 (Southwestern) & $6(9.2 \%)$ & $27(17.1 \%)$ & & $33(14.8 \%)$ \\
\hline Location in metropolitan region & $46(70.8 \%)$ & $105(66.5 \%)$ & 0.53 & $151(67.7 \%)$ \\
\hline \multicolumn{5}{|l|}{ County-level characteristics } \\
\hline Percent poverty & $18.2(5.2)$ & $19.1(5.9)$ & 0.25 & $18.9(5.7)$ \\
\hline Percent uninsured & $13.9(5.4)$ & $11.9(3.4)$ & 0.0008 & $12.5(4.1)$ \\
\hline Percent with high school education & $83.2(6.6)$ & $84.6(5.5)$ & 0.13 & $84.2(5.8)$ \\
\hline Located in urban area & $46(70.7 \%)$ & $105(66.5 \%)$ & 0.53 & $151(66.7 \%)$ \\
\hline Median income, in the USA Dollars & $47,084(10,471)$ & $45,590(10,652)$ & 0.34 & $46,026(10,598)$ \\
\hline Percent White & $69.9(20.7)$ & $69.8(20.1)$ & 0.98 & $69.9(20.2)$ \\
\hline Percent Hispanic & $10.9(14.6)$ & $8.6(12.9)$ & 0.23 & $9.3(13.4)$ \\
\hline
\end{tabular}

All statistics were calculated using unpaired t tests, chi-square tests, or Fisher's exact tests, where appropriate

Italicized values: $p<0.05$ 


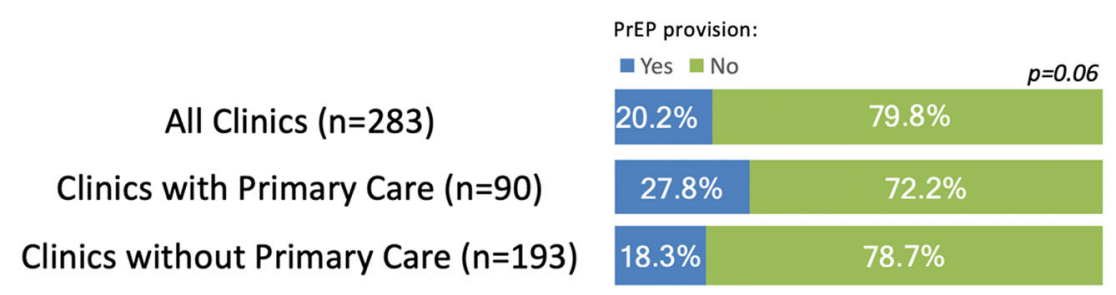

Figure 1 PrEP provision overall and by primary care provision status.

currently providing PrEP was greater among clinics that also provided primary care services compared to those that did not, though the difference was just outside of standard levels for statistical significance $(27.8 \%$ vs. $18.3 \%, p=0.06$; Fig. 1). To further explore other factors that may explain higher PrEP provision in primary care-providing clinics, we compared clinic- and county-level characteristics by primary care status (Table 1). More primary care-providing clinics reported their clinic type as FQHCs, while more of the nonprimary care-providing clinics were health departmentbased clinics $(p<0.0001)$. Primary care-providing clinics more commonly had insurance navigation support onsite $(p$ $=0.019)$, but they also had a higher percentage of people in their county who were uninsured $(p=0.0008)$. There were no other differences in county-level sociodemographic characteristics between clinics that did and did not provide primary care.
Perceptions of Cost and Available Resources in Respondents from Non-PrEP Clinics. Among the 414 survey respondents who were practicing in non-PrEP-providing clinics, those in clinics with primary care services were more likely to respond that they had the necessary support in terms of budget or financial resources $(p<0.01)$ and staffing $(p<0.01)$ for PrEP implementation compared to those without primary care services (Table 2), but did not differ in other survey items.

However, there were no significant differences in any of the specific clinic staffing or facilities variables relevant for PrEP delivery by provision of primary care services: there was no significant difference in the respondent's ability to write prescriptions $(34.5 \%$ in primary care vs. $43.0 \%$ in non-primary care, $p=0.09)$, the availability of onsite staff for insurance navigation $(71.8 \%$ in primary care vs. $64.3 \%$ in non-primary care, $p=0.12)$, or the presence of onsite pharmacies $(51.4 \%$ in primary care vs. $44.6 \%$ in non-primary care, $p=0.19$ ).

Table 2 Cost- and Resource-Related Consolidated Framework for Implementation Research (CFIR) Survey Results Among Respondents from Non-PrEP Clinics, by Provision of Primary Care Services $(n=414)$

\begin{tabular}{|c|c|c|c|c|c|c|}
\hline Variable & $\begin{array}{l}\text { Primary care: } \\
\text { mean (SD) }\end{array}$ & $\begin{array}{l}\text { No primary care: } \\
\text { mean (SD) }\end{array}$ & $\begin{array}{l}\text { Parameter } \\
\text { estimate }(\beta)\end{array}$ & $\begin{array}{l}\text { Standard } \\
\text { error }\end{array}$ & $t$ value & $p$ value $^{\mathrm{a}}$ \\
\hline \multicolumn{7}{|l|}{ Domain: cost } \\
\hline $\begin{array}{l}\text { 1. Providing PrEP at my clinic requires too } \\
\text { many human resources. }\end{array}$ & $2.92(0.87)$ & $2.96(0.88)$ & $0.0098(0.027)$ & 0.091 & -0.37 & 0.72 \\
\hline 2. PrEP is too expensive. & $3.31(0.79)$ & $3.26(0.92)$ & $-0.015(0.027)$ & 0.091 & 0.54 & 0.59 \\
\hline $\begin{array}{l}\text { 3. My clinic would only pay for PrEP care } \\
\text { (e.g., labs and visits only) if a patient } \\
\text { at my clinic cannot pay for PrEP care. }\end{array}$ & $2.60(0.90)$ & $2.53(0.97)$ & $-0.018(0.025)$ & 0.099 & 0.72 & 0.47 \\
\hline $\begin{array}{l}\text { 4. My clinic would only be interested } \\
\text { in adopting PrEP if funding was provided } \\
\text { for PrEP care. }\end{array}$ & $3.62(0.78)$ & $3.66(0.84)$ & $0.012(0.029)$ & 0.086 & -0.42 & 0.67 \\
\hline $\begin{array}{l}\text { 5. Uncertainty around continuation of federal } \\
\text { funding (e.g., Title X, Medicaid, Ryan } \\
\text { White) would prohibit our clinic from } \\
\text { adopting PrEP. }\end{array}$ & $3.12(0.76)$ & $3.19(0.84)$ & $0.011(0.029)$ & 0.085 & -0.39 & 0.70 \\
\hline Average of cost domain & $3.27(0.51)$ & $3.30(0.57)$ & $0.019(0.043)$ & 0.055 & -0.45 & 0.65 \\
\hline \multicolumn{7}{|l|}{ Domain: PrEP-specific funding resources } \\
\hline $\begin{array}{l}\text { 1. My clinic would only be interested } \\
\text { in adopting PrEP if Title X or other } \\
\text { funding sources required it. }\end{array}$ & $3.26(0.81)$ & $3.39(0.86)$ & $0.040(0.022)$ & 0.088 & -1.42 & 0.16 \\
\hline \multicolumn{7}{|l|}{ Domain: general assessment of resources } \\
\hline $\begin{array}{l}\text { 1. We have the necessary support in terms } \\
\text { of budget or financial resources. }\end{array}$ & $2.69(0.94)$ & $2.39(1.05)$ & $-0.064(0.024)$ & 0.11 & 2.73 & 0.0067 \\
\hline $\begin{array}{l}\text { 2. We have the necessary support in terms } \\
\text { of training. }\end{array}$ & $3.22(0.96)$ & 3.09 (1.07) & $-0.028(0.024)$ & 0.11 & 1.17 & 0.24 \\
\hline $\begin{array}{l}\text { 3. We have the necessary support in terms } \\
\text { of facilities. }\end{array}$ & $3.26(0.91)$ & 3.07 (1.08) & $-0.041(0.024)$ & 0.11 & 1.72 & 0.086 \\
\hline $\begin{array}{l}\text { 4. We have the necessary support in terms } \\
\text { of staffing. }\end{array}$ & $3.05(0.95)$ & $2.67(1.14)$ & $-0.071(0.022)$ & 0.12 & 3.20 & 0.0015 \\
\hline
\end{tabular}


Table 3 Descriptive Statistics of Interview Participants from NonPrEP-Providing Clinics, Stratified by Provision of Primary Care Services $(n=32)$

\begin{tabular}{lll}
\hline \hline Demographic variable & \multicolumn{2}{l}{ Primary care provision status } \\
\cline { 2 - 3 } & $\begin{array}{l}\text { Primary care } \\
(\boldsymbol{n}=\mathbf{9 )}\end{array}$ & $\begin{array}{c}\text { No primary } \\
\text { care }(\boldsymbol{n}=\mathbf{2 3})\end{array}$ \\
\hline $\begin{array}{lll}\text { Respondent-level characteristics } \\
\text { Clinic role: }\end{array}$ & \\
Provider & $5(55.6 \%)$ & $15(65.2 \%)$ \\
Administrator & $4(44.4 \%)$ & $8(34.8 \%)$ \\
& & \\
Clinic-level characteristics & & \\
Clinic classification & & \\
Federally qualified health center & $7(77.8 \%)$ & $4(17.4 \%)$ \\
Health department-based clinic & $1(14.3 \%)$ & $19(82.6 \%)$ \\
Other family planning clinic type & $1(14.3 \%)$ & $1(4.3 \%)$ \\
Department of Health and Human Services region: & \\
Region 3 (mid-Atlantic) & $1(11.1 \%)$ & $9(39.1 \%)$ \\
Region 4 (Southeastern) & $8(88.9 \%)$ & $8(34.8 \%)$ \\
Region 6 (Southwestern) & $0(0.0 \%)$ & $6(26.1 \%)$ \\
\hline
\end{tabular}

\section{Qualitative Results}

Of the 56 total interview participants, 32 were from clinics that did not provide PrEP and were included in this analysis, as to better understand barriers to implementation in this setting. The demographics of interview participants from non-PrEP-providing clinics are described in Table 3. Non-PrEP, Non-Primary Care Clinics. Respondents from clinics who did not provide PrEP and did not provide concomitant primary care services nearly ubiquitously cited cost of labs, especially hepatitis serologies and renal function tests, as a substantial budget or financial resource concern surrounding potential PrEP implementation:

We don't test for Hepatitis B or C and we don't do any kidney function tests... it's just a matter of the cost for these tests. (Clinical provider)

A lack of overall staffing, and specifically staffing to help with PrEP-related insurance navigation or patient assistance programs, also was frequently mentioned by respondents. More training around PrEP cost, waivers, and patient assistance programs was often desired.

... We do have a social worker here now who's kind of overwhelmed with a number of things to address in any given day, but if we had staff who could help clients... who are insurance eligible to help them complete applications and get their paperwork together... (Administrator)

So that would be like having the social worker like really know how to, you know, what programs in [STATE] will pay for this depending on what their insurance is and what the options are if they don't have insurance. I mean we do that like with patients that don't have insurance. We help them fill out forms to get like breast cancer screening and there's some programs paid for by the state actually is like from the tobacco fund from when they sued tobacco companies. (Clinical provider)

Two providers described their only prior experiences with patient assistance programs for various birth control methods, and how burdensome the paperwork was without having specialized staff to assist with the process.

Yeah there's medication assistance programs but then you're spending all your time doing all that paperwork and as nurse practitioners we really aren't - when we have other things going on we don't really want to be sitting there fill out prior authorizations and doing medication assistance programs. They want us to do the one for Mirena, the [PAYER ASSISTANCE PROGRAM], but honestly it takes so much time it's easier for us to just give them Mirena and be like, 'Don't worry. The grant will take care of it.' (Clinical provider)

Staffing the frequent follow-up visits every 3 months was also commonly cited concern.

We currently do not have the staff capacity to be a PrEP site based on the labs and follow ups that are required. (Administrator)

[The biggest concern is] to have the staff to probably encourage the people to return every 3 months for their follow-up because sometimes that can take effort. They may need a letter, they may need a telephone call... (Clinical provider)

Overall, the cost of individual lab costs associated with PrEP initiation and follow-up monitoring, as well as the required staffing for PrEP insurance navigation and follow-up visits, was the most commonly cited cost- and resource-related concerns regarding PrEP implementation in this population.

Non-PrEP, Primary Care Clinics. Different themes surrounding financial and staffing resources for PrEP implementation emerged in the interviews of respondents from non-PrEP providing clinics that did provide primary care services. Whereas respondents from clinics who did not provide primary care services commonly cited concerns about the cost of laboratory tests associated with PrEP screening and monitoring, none of the respondents from clinics who provided primary care cited any concerns about these tests - they routinely gather them and there would be no significant perceived financial burden associated with their individual costs. 
Rather, these respondents cited concerns about cumulative cost of these labs that must be collected every 3 months, likening the financial burden to conditions like hypertension and diabetes:

Lab work can be - even at low-contracted prices - can be expensive for clients. For example we have patients that have hypertension and diabetes that need chemistries done, Hemoglobin A1C, every three to six months, and we have a hard time getting those people that have these types of conditions coming in and getting their lab work done every six months as needed. Or as recommended. Because of cost. And a lot of those labs are very inexpensive. The general lab for a diabetic patient runs about $\$ 25$ to $\$ 35$ - something that if they went to an outside lab and got would be $\$ 200$ to $\$ 300$ - and they still can’t afford that. (Administrator/ Center coordinator)

But over the long term and the return visits and the lab repeating those labs, sometimes the labs get to be costly. So like I said before, I wouldn't want to start a patient on PrEP and say oh, you need this lab test in six months and it will be $\$ 50$. And they say, “I don't have $\$ 50$ ". I wouldn't want to start them then run into that problem. So then I would factor in the cost of the additional labs because right now, the labs I do, I'm saying like people who have blood pressure or diabetes. (Clinical provider)

Regarding affordability of PrEP and patient assistance programs, respondents cited that they could rely on financial counselors or onsite insurance navigators that were part of the adult primary care part of their practice:

In our Health Department, we have adult primary care, so we do have a current staff that deal with and assist adult clients with prescription assistance... So the prescription assistance program does help clients with things like blood pressure medicine, diabetes medicines and stuff like that, so I'm assuming this might be similar. (Administrator/Center coordinator)

Further, respondents noted that they could utilize preexisting clinic structure and staff that is already able to handle management of patients with chronic medical conditions to manage the quarter annual follow-up visits required for PrEP.

I mean, it can't be more complicated than treating somebody with diabetes. I mean, seriously, when you're talking about needing to do very specific diet and exercise and medication regimen and follow up and lab and all that. I would say it would be very similar. (Administrator/Center coordinator)

The provider would be able to prescribe to those patients and arrange for a follow-up appointment every three months. You know, our provider, as well as our front desk, our entity staff would be able to do [this]. I would say [the concern of providing these services on top of the current existing schedule] maybe minimal because I think it would be, again, integrated into, you know, our current process. It would just be a different type of visit. (Clinical provider)

One provider compared potential PrEP implementation to a medication-assisted treatment of opioid use disorder their clinic had previously initiated and described how similar the implementation infrastructures could be.

Overall, in contrast to the cost- and resource-related concerns of non-PrEP, non-primary care clinics, the clinics with concomitant primary care services instead had concerns about the cumulative, not individual, cost of labs, and had much fewer concerns about staffing PrEP insurance navigation and follow-up visits.

\section{DISCUSSION}

This study demonstrates that although PrEP delivery in Title $\mathrm{X}$-funded family planning clinics in the Southern US is limited, with only one-fifth of all clinics providing PrEP, clinics with co-located primary care services more commonly provide PrEP and report fewer perceived cost and resource barriers. PrEP use has been associated with an increased uptake of nonPrEP-related healthcare like the influenza vaccine, tobacco screening, depression screening, and glucose testing among men who have sex with men, suggesting that there may be synergy in the provision of PrEP and other routine primary care services. ${ }^{20}$ Our findings further support this potential synergy and the expansion of PrEP in women's health settings, particularly those that already provide other primary care services. These findings are aligned also with the U.S. Department of Health and Human Services "Ending the HIV Epidemic" initiative, announced in February 2019, which emphasizes the role of existing healthcare systems in reducing new HIV infections. ${ }^{21}$ The connection between PrEP provision and primary care services within family planning clinics is especially important for women, as many women rely on family planning clinics for their usual or exclusive healthcare source. $^{22,} 23$

Our analysis demonstrated that FQHCs or other community-based health clinics were more likely to offer colocated primary care services as compared to other family planning clinic types. FQHCs are funded by the Health 
Resources and Services Administration (HRSA) as community-based healthcare providers that provide primary care services in underserved areas on a sliding fee scale based on ability to pay. ${ }^{24}$ Focus groups with clinic staff from FQHCs in South Florida found that providers had universally heard of PrEP and were able to describe the recommended population to receive PrEP, and that they felt PrEP implementation was feasible at their site. ${ }^{25}$ However, despite centers providing care at no or low cost on a sliding scale, concerns of cost and insurance were still identified as significant barriers to PrEP care. Training and technical assistance around associated costs of PrEP may be one way to at least address unfavorable cost perceptions, though other interventions will be needed to address other barriers to PrEP care.

This analysis provides insight into drivers of higher perceived resource barriers to PrEP integration in Title X clinics that do not provide concomitant primary care. Among the general measures of resources surveyed, clinics without co-located primary care reported significantly lower scores in facilities- and staffing-related resources, but not in financial- or training-related resources. Family planning clinics with concomitant primary care services likely have different clinical and operational infrastructure to provide the recommended PrEP laboratory testing and longitudinal care. ${ }^{26}$ This infrastructure may lend itself to easier integration of all steps of PrEP delivery and therefore more PrEP provision overall, and suggests that more robust facilities and staffing resources may be necessary for non-primary care providing clinics. On the other hand, since training has been previously noted by family planning providers as a barrier to PrEP delivery, ${ }^{18}$ Title $\mathrm{X}$ clinics may benefit from additional PrEP training and educational resources irrespective of primary care infrastructure. Future research could explore those trainings impact on later adoption of PrEP implementation in the clinic.

Several major themes from the qualitative analysis can further inform strategies for PrEP implementation across Title X clinics in the South. Respondents from primary care-providing family planning clinics were more comfortable with the longitudinal lab monitoring that is required for PrEP, as frequent labs are integral to the management of chronic conditions seen in the primary care setting. Further, family planning clinics with concomitant primary care services were confident that they could manage the costs associated with PrEP care via financial counselors or onsite insurance navigators that were already part of their practice. Lastly, respondents from clinics with primary care services stated that their clinic already had the infrastructure to support the scheduling and visit infrastructure for follow-up visits included in PrEP care and monitoring. These results suggest that Title $\mathrm{X}$ clinics with concomitant primary care may be key avenues for immediate integration of PrEP. On the other hand, those without primary care services may need additional support for longitudinal care and monitoring before being ready to fully implement PrEP services, along with strengthening of linkages to nearby PrEPproviding clinics as an alternative strategy to increasing PrEP access for their patients.

Our study had several limitations. First, it was a convenience sample of those who agreed to and completed key components of the web-based survey. Further, we had limited respondents from certain clinic types (for example, PrEPproviding non-metropolitan clinics), limiting our ability to describe their characteristics. Further, provider and administrator perceptions were utilized for analyses rather than direct observations. Finally, the study was conducted among staff of Title X-funded family planning clinics, and therefore, findings may not be generalizable across other women's health settings. Nonetheless, a key strength of this study was the diversity in geographic location and clinic characteristics among the clinics represented by study participants, as well as the mixed methods design that allowed for richer contextualization of barriers and facilitators of PrEP provision in those settings.

In conclusion, among Title $\mathrm{X}$ family planning clinics in the Southern US, current PrEP provision was higher among clinics with co-located primary care services. Among clinics not currently providing PrEP, those that also provide primary care services have lower perceived cost and resource barriers and therefore may be key avenues for early expansion of PrEP delivery for women. These findings underscore the importance of further investigating and addressing resource-related and other barriers to PrEP implementation across family planning clinics in high HIV-burden areas, as to better reach all individuals at risk for HIV infection, including women.

Acknowledgments: We acknowledge the contributions of Ms. Ashley Phillips and Ms. Ilyssa Tamler in survey development and dissemination. We also thank the coordinating center of the Adolescent Trials Network for overall study support and data management.

Corresponding Author: Anandi N. Sheth, MD; Grady Health System, Atlanta, GA, USA (e-mail: ansheth@emory.edu).

Author Contributions Caroline Coleman developed the research question, summarized the appropriate variables in this analysis, performed all statistical analyses, and wrote the paper under the supervision of Dr. Sheth. Drs. Sheth, Escoffery, and Sales designed the study and data collection instruments, developed the research question, supervised all statistical analyses, and edited the paper. Ms. Powell and Ms. Piper conducted the key informant interviews and codified them in NVivo and reviewed the final version of the paper.

Funding This study was funded by the National Institutes of Health Adolescent Trials Network (NICHD U24HD089880 to LaVange/ Carpenter/Hudgens, subaward to Sales/Sheth).

\section{Compliance with ethical standards:}

Conflict of Interest: Drs. Sheth and Sales report grants from Gilead Sciences, Inc, outside the submitted work. No other authors have anything to disclose. 


\section{REFERENCES}

1. Centers for Disease Control and Prevention. CDC Issue Brief: HIV in the Southern United States 2016 [updated September 2019]. Available from: https://www.cdc.gov/hiv/pdf/policies/cdc-hiv-in-the-south-issue-brief. pdf. Accessed 11 Nov 2019

2. Centers for Disease Control and Prevention. HIV Among Women 2018 [updated January 20, 2020]. Available from: www.cdc.gov/hiv/group/ gender/women/index.html.. Accessed 11 Nov 2019.

3. Thomson KA JMB, Nelly R. Mugo, Linda-Gail Bekker, Connie L. Celum, Renee Heffron. Tenofovir-based Oral PrEP Prevents HIV Infection among Women. Curr Opin HIV AIDS 2016;11(1):18-26. https://doi. org/10.1097/COH.0000000000000207

4. Auerbach JD, Kinsky S, Brown G, et al. Knowledge, attitudes, and likelihood of pre-exposure prophylaxis (PrEP) use among US women at risk of acquiring HIV. AIDS Patient Care STDS 2015;29(2):102-10. https://doi.org/10.1089/apc.2014.0142 [published Online First: 2014/12/17]

5. Siegler AJ, Mouhanna F, Giler RM, et al. The prevalence of preexposure prophylaxis use and the pre-exposure prophylaxis-to-need ratio in the fourth quarter of 2017, United States. Ann Epidemiol 2018;28(12):841-49. https://doi.org/10.1016/j.annepidem.2018.06. 005 [published Online First: 2018/07/10]

6. Patel AS, Goparaju L, Sales JM, et al. Brief Report: PrEP Eligibility Among At-Risk Women in the Southern United States: Associated Factors, Awareness, and Acceptability. J Acquir Immune Defic Syndr 2019;80(5):527-32. https://doi.org/10.1097/QAI.0000000000001950 [published Online First: 2019/01/17]

7. Stormo AR, Saraiya M, Hing E, et al. Women's clinical preventive services in the United States: who is doing what? JAMA Intern Med 2014;174(9):1512-4. https://doi.org/10.1001/jamainternmed.2014. 3003 [published Online First: 2014/07/09]

8. Seidman $\mathbf{D}$, Carlson $\mathbf{K}$, Weber $\mathbf{S}$, et al. United States family planning providers' knowledge of and attitudes towards preexposure prophylaxis for HIV prevention: a national survey. Contraception 2016;93(5):463-9. https://doi.org/10.1016/j.contraception.2015.12.018 [published Online First: 2016/01/17]

9. Blumenthal J, Jain S, Krakower D, et al. Knowledge is Power! Increased Provider Knowledge Scores Regarding Pre-exposure Prophylaxis (PrEP) are Associated with Higher Rates of PrEP Prescription and Future Intent to Prescribe PrEP. AIDS Behav 2015;19(5):802-10. doi: https://doi.org/10.1007/s10461-015-0996-z [published Online First: 2015/01/27]

10. Patel RR, Mena $\mathbf{L}$, Nunn A, et al. Impact of insurance coverage on utilization of pre-exposure prophylaxis for HIV prevention. PLoS One 2017;12(5):e0178737. https://doi.org/10.1371/journal.pone.0178737 [published Online First: 2017/05/31]

11. Jones RK, Sonfield A. Health insurance coverage among women of reproductive age before and after implementation of the affordable care act. Contraception 2016;93(5):386-91. https://doi.org/10.1016/j.contraception.2016.01.003 [published Online First: 2016/01/24]

12. Fowler CI, Gable J, Wang J, Lasater B. Family Planning Annual Report: 2018 national summary: RTI International; 2018. Available from: https://opa.hhs.gov/sites/default/files/2020-07/title-x-fpar-2018-national-summary.pdf. Accessed 25 Oct 2020.

13. Dawson R. Trump Administration's Domestic Gag Rule Has Slashed the Title X Network's Capacity by Half: Guttmacher Institute, 2020.
14. Sales JM, Escoffery C, Hussen SA, Haddad LB, McCumber M, Kwiatkowski E, Filipowicz T, Sanchez M, Psioda M, Sheth AN. Preexposure prophylaxis implementation in family planning services across the Southern United States: findings from a survey among staff, providers, and administrators working in Title X-funded clinics. AIDS and Behavior 2020 (in press).

15. Sales JM, Escoffery C, Hussen SA, et al. Pre-Exposure Prophylaxis Integration into Family Planning Services at Title $\mathrm{X}$ Clinics in the Southeastern United States: A Geographically-Targeted Mixed Methods Study (Phase 1 ATN 155). JMIR Res Protoc 2019;8(6):e12774. https://doi. org/10.2196/12774 [published Online First: 2019/06/15]

16. Piper KN, Escoffery C, Sales JM, Sheth AN. Models of HIV pre-exposure prophylaxis care used in Title X family planning clinics in the Southern United States. Journal of Adolescent Health 2020; https://doi.org/10. 1016/j.jadohealth.2020.10.005 [published Online First 2020/11/5].

17. CFIR Research Team. Consolidated Framework for Implementation Research (CFIR) Technical Assistance Website Qualitative Data Guide. 2019 [Available from: https://cfirguide.org/. Accessed 11 Nov 2019.

18. AIDSVu (aidsvu.org). Emory University, Rollins School of Public Health. Deeper Look: PrEP 2019. Available from: https://aidsvu.org/ resources/deeper-look-prep/. Accessed 11 Nov 2019.

19. Miles MB, Huberman AM, Saldana J. Qualitative data analysis : a methods sourcebook. Third edition. SAGE Publications, 2013.

20. Marcus JL, Levine K, Grasso C, et al. HIV Preexposure Prophylaxis as a Gateway to Primary Care. Am J Public Health 2018;108(10):1418-20. https://doi.org/10.2105/AJPH.2018.304561 [published Online First: 2018/07/20]

21. Office of Infectious Disease and HIV/AIDS Policy, HHS. "What is 'Ending the HIV Epidemic: A Plan for America'?" 2019. Available from: https:// www.hiv.gov/federal-response/ending-the-hiv-epidemic/overview. Accessed 11 Nov 2019.

22. Gold RB SA, Richards CL, Frost JJ. Next Steps for America's Family Planning Program: Leveraging the Potential of Medicaid and Title X in an Evolving Health Care System: Guttmacher Institute; 2009. Available from: https://www.guttmacher.org/sites/default/files/ pdfs/pubs/summaries/NextStepsExec.pdf. Accessed 11 Nov 2019.

23. Sugerman $\mathbf{S}$, Halfon $\mathbf{N}$, Fink A, et al. Family planning clinic patients: their usual health care providers, insurance status, and implications for managed care. J Adolesc Health 2000;27(1):25-33. https://doi.org/10. 1016/s1054-139x(99)00126-3 [published Online First: 2000/06/27]

24. Health Resources and Services Administration (HRSA). Federally Qualified Health Centers Eligibility 2018 [updated May 2018. Available from: https://www.hrsa.gov/opa/eligibility-and-registration/ health-centers/fqhc/index.html. Accessed 11 Nov 2019.

25. Doblecki-Lewis S, Jones D. Community Federally Qualified Health Centers as Homes for HIV Preexposure Prophylaxis: Perspectives from South Florida. J Int Assoc Provid AIDS Care 2016;15(6):522-28. https://doi.org/ 10.1177/2325957416661422 [published Online First: 2016/08/10]

26. Marcus JL, Volk JE, Pinder J, et al. Successful Implementation of HIV Preexposure Prophylaxis: Lessons Learned From Three Clinical Settings. Curr HIV/AIDS Rep 2016;13(2):116-24. https://doi.org/10.1007/ s11904-016-0308-x [published Online First: 2016/02/24]

Publisher's Note: Springer Nature remains neutral with regard to jurisdictional claims in published maps and institutional affiliations. 